\title{
HEREDITARY PROPERTIES OF DIRECT SUMMANDS OF ALGEBRAS
}

\author{
Hanspeter Kraft, Lance W. Small, Nolan R. Wallach
}

\begin{abstract}
We consider subrings $S$ of rings $R$ such that $R=S \oplus V$ with $V$ either two sided invariant under multiplication by $S$ or invariant under the commutator with $S$. We show that some important properties of $R$ are inherited by $S$ under such conditions. One is the FCR-property which says that every finite dimensional representation is completely reducible. Another application gives a characterization (in characteristic zero) of reductive subgroups of reductive groups.
\end{abstract}

\section{Introduction}

Enveloping algebras of finite dimensional semisimple Lie algebras (in characteristic zero) possess the remarkable property that their finite dimensional representations are completely reducible. We call this the FCR-property (see §3). Are there other wide classes of algebras with this property? In [KrS94] it was shown that the fixed rings of actions of finite group on such FCR-algebras are also FCR (see Proposition 3). Additionally, it was shown that fixed rings of such actions on enveloping algebras yielded really "new" examples.

In general, if $R$ is a ring and $H$ a finite group of automorphisms of $R$ with the order of $H$ invertible in $R$, then the fixed point $\operatorname{ring} R^{H}$ is a direct summand of $R$ as an $R^{H}$-bimodule. Thus, it is reasonable to ask whether the FCR-property of a ring $R$ is inherited by a subring $A$ such that $R$ is a finitely generated (left) $A$-module and $R=A \oplus V$ where $V$ is an $A$-bimodule. The answer is yes (Theorem 3 ), and this theorem is an easy generalization of an elementary result which says that a subring $A$ of a semisimple ring is semisimple if it admits a complement which is left and right stable under $A$ (Theorem 1). In fact, it turned out that in characteristic $\neq 2$ it suffices to assume that the complement is stable under commutation with $A$ (Proposition 1).

These results are consequences of what we call the "Main Lemma" (and its proof) which is a criterion for the vanishing of the nilradical of a Lie subring of a semisimple ring. The Main Lemma also implies some important properties of closed subgroups of reductive linear algebraic groups.

Received February 16, 1999. 


\section{$\S 1$. Direct summands of semisimple rings}

In this paper all rings $R$ contain a unit element $1=1_{R}$. As usual, we call a ring $R$ semisimple if $R$, considered as a left $R$-module is semisimple (cf. [Pi82, $3.1]$ ). Also we will use the notation $[a, b]=a b-b a$ for the commutator of two elements in a ring. A $\mathbb{Z}$-submodule $L$ of a ring $R$ is called a Lie subring if $L$ is closed under commutation, $[L, L] \subset L$. Finally, a $\mathbb{Z}$-submodule $N \subset L$ is called an ideal if $[L, N] \subset N$.

Main Lemma. Let $R$ be a semisimple ring and assume that 2 is invertible in $R$. Let $L \subset R$ be a Lie subring such that $R=L \oplus V$ where $V$ is a $\mathbb{Z}$-submodule with $[L, V] \subset V$. Then every ideal of $L$ consisting of nilpotent elements is trivial.

Proof. By Wedderburn's Theorem ([Pi82, 3.5]), $R$ is a finite direct sum of matrix algebras over division rings:

$$
R=M_{n_{1}}\left(D_{1}\right) \oplus M_{n_{2}}\left(D_{2}\right) \oplus \cdots \oplus M_{n_{s}}\left(D_{s}\right) .
$$

Let $N \subset L$ be an ideal consisting of nilpotent elements and let $x \in N$. Then there exist $y, h \in R$ such that $[x, y]=h$ and $[h, x]=2 x$. In fact, this is a direct consequence of the Jordan normal form for nilpotent matrices in matrix rings over division algebras applied to each component $x_{i} \in M_{n_{i}}\left(D_{i}\right)$ of $x$.

Now write $h=h_{L}+h_{V}, y=y_{L}+y_{V}$ where $h_{L}, y_{L} \in L$ and $h_{V}, y_{V} \in V$. Then the assumption on the subspace $V$ implies that $\left[x, y_{L}\right]=h_{L}$ and $\left[h_{L}, x\right]=2 x$. In particular, $h_{L} \in N$ which implies that $h_{L}$ is nilpotent. So $\operatorname{ad}\left(h_{L}\right)$ is nilpotent on $R$. Since $\operatorname{ad}\left(h_{L}\right) x=2 x$ we get $x=0$.

The first application is the following proposition. Recall that a semisimple ring is always (left) Artinian, i.e., it satisfies the descending chain condition for left ideals. We will denote this condition by "dcc".

Proposition 1. Let $A$ be a subring of a semisimple ring $R$. Assume that 2 is invertible in $R$ and that $R=A \oplus V$ where $V$ is a $\mathbb{Z}$-submodule with $[A, V] \subset V$. Then every nilpotent ideal of $A$ is trivial. In particular, if $A$ satisfies dcc, then A is semisimple.

Proof. The first statement is an immediate consequence of the Main Lemma. The second statement follows from the first since for every (left) Artinian ring $A$ the radical $J \subset A$ is nilpotent and $A / J$ is semisimple (see [Pi82, 3.1 Proposition a and 4.4 Proposition]).

We do not know if Proposition 1 also holds in characteristic 2. However, the following result allows us to say something in the associative situation. We will give two proofs. The first one is in the spirit of the proof of the Main Lemma, the second is due to Guralnick and uses different methods.

Let us point out that this theorem can also be obtained from results in [Be71] (see proof of Proposition 1.2), although it is not stated explicitly. 
Theorem 1. Let $A$ be a subring of a semisimple ring $R$ such that $R=A \oplus V$ as an A-bimodule. Then $A$ is also semisimple.

First proof. If $I$ is an ideal of $A$ then $R I=I \oplus V I$ which implies that $R I \cap A=I$. Thus $A$ satisfies dcc. Therefore, it suffices to prove that the radical $N$ of $A$ is zero. For that purpose it is enough to show that if $x \in N$ and $x^{2}=0$ then $x=0$.

As before, we use the decomposition

$$
R=M_{n_{1}}\left(D_{1}\right) \oplus M_{n_{2}}\left(D_{2}\right) \oplus \cdots \oplus M_{n_{s}}\left(D_{s}\right)
$$

to put each component $x_{i}$ of $x$ into Jordan normal form. Since $x^{2}=0$ we see that the Jordan blocks have at most size $2 \times 2$. Let $y \in R$ be the "transpose" of $x$, i.e., $y=\sum x_{i}^{t}$, and set $h:=x y$. A simple calculation shows that $h x=x$. As before we write $y=y_{A}+y_{V}$ with $y_{A} \in A$ and $y_{V} \in V$. Then $h=x y_{A}+x y_{V}$ with $x y_{A} \in A$ and $x y_{V} \in V$. Set $h_{A}=x y_{A}$ and $h_{V}=x y_{V}$. Then $h_{A} x \in A$ and $h_{V} x \in V$ and so $x=h x=h_{A} x$. But $h_{A}=x y_{a} \in N$ and therefore $\left(h_{A}\right)^{k}=0$ for some $k$. It follows that $x=\left(h_{A}\right)^{k} x=0$.

Second proof (GURALNICK). It is a direct consequence of the definition that an algebra is semisimple if and only if every left ideal is a direct summand. Let $I$ be a left ideal of $A$. It follows that $R I=I \oplus V I$ and so $I$ is a direct summand of $R I$ as a left $A$-module. By assumption, $R I$ is a direct summand of $R$ as an $R$-module. Hence, the left ideal $I$ is a direct summand of $A$.

As a corollary we recover an old theorem of Levitzki (see [Mo80, Chap. 1, Theorem 1.15]).

Corollary 1. If $R$ is a semisimple ring and $H$ a finite group of automorphism of $R$ such that the order $|G|$ is invertible in $R$, then the fixed point ring $R^{H}$ is also semisimple.

\section{$\S 2$. Applications to reductive groups}

We can look upon Proposition 1 and Theorem 1 above as the assertion that under a certain hypothesis the invertible elements in a subalgebra $A$ of the matrix ring $M_{n}(K)$ over a field $K$ form a reductive affine algebraic group. That result has the following generalization.

Theorem 2. Let $G$ be a (Zariski-)closed subgroup of $\mathrm{GL}_{n}(K)$ where $K$ is an algebraically closed field of characteristic not equal to 2. If $M_{n}(K)=\operatorname{Lie}(G) \oplus V$ such that $[\operatorname{Lie}(G), V] \subset V$, then $G$ is reductive.

Proof. Let $R_{u}(G)$ be the unipotent radical of $G$. Putting $L:=\operatorname{Lie}(G)$ and $N:=\operatorname{Lie}\left(R_{u}(G)\right)$, then $N$ is a nilpotent ideal and the hypotheses of the Main Lemma are satisfied. Thus $N=(0)$ and so $R_{u}(G)=\{1\}$.

In characteristic 0 , the above theorem gives a characterization of closed reductive subgroups of a reductive affine algebraic group. 
Corollary 2. Let $G$ be a reductive affine algebraic group over an algebraically closed field of characteristic 0 and let $H \subset G$ be a (Zariski-)closed subgroup. Then $H$ is reductive if and only if $\operatorname{Lie}(H)$ has an $\operatorname{Ad}(H)$-stable complement in $\operatorname{Lie}(G)$.

This follows since the notions of reductive algebraic group and linearly reductive algebraic group coincide in characteristic 0 . This means that every finite dimensional representation of a reductive algebraic group in characteristic zero is completely reducible.

There is a partial converse to Theorem 2 above, valid in "good characteristic".

Proposition 2. Assume that the characteristic of $K$ is different from 2,3 and 5. Then a connected affine algebraic group $G$ is reductive if and only if there exists a group $G^{\prime}$ isogenous to $G$ and a closed embedding $G^{\prime} \subset \mathrm{GL}_{n}(K)$ (for some $n)$ such that $\operatorname{Lie}\left(G^{\prime}\right)$ admits an $\operatorname{Ad}\left(G^{\prime}\right)$-stable complement in $M_{n}(K)$.

Proof. In light of the above we need only to show that if $G$ is reductive then such an isogeny exists. For a simple group $G$ this is the content of Proposition 1 (p. 104) in [St74]. In general, we use the well-known fact that a connected reductive group is isogenous to a product of simple groups and a torus.

The following result (in characteristic 0) is one of Cartan's criteria.

Corollary 3. Let $K$ be a field with $\operatorname{char}(K) \neq 2$. If $\mathfrak{g}$ is a Lie subalgebra of $M_{n}(K)$ such that the form $B(X, Y):=\operatorname{tr}(X Y)$ is non-degenerate on $\mathfrak{g}$ then every nilpotent ideal of $\mathfrak{g}$ is trivial. In particular, if $G \subset \mathrm{GL}_{n}(K)$ is a (Zariski-)closed subgroup such that the form $B$ restricted to Lie $G$ is non-degenerate then $G$ is reductive.

Proof. Set $V:=\left\{Y \in M_{n}(K) \mid B(Y, X)=0\right.$ for all $\left.X \in \mathfrak{g}\right\}$. Then $[\mathfrak{g}, V] \subset V$ and $V \cap \mathfrak{g}=(0)$. Thus the Main Lemma of $\S 1$ implies the result.

\section{$\S 3$. The FCR-property for direct summands}

It is well-known that the enveloping algebra $\mathfrak{U}(\mathfrak{g})$ of a semisimple Lie algebra $\mathfrak{g}$ in characteristic zero has the following two important properties:

- Every finite dimensional representation of $\mathfrak{U}(\mathfrak{g})$ is completely reducible.

- $\mathfrak{U}(\mathfrak{g})$ is residually finite dimensional, i.e., the intersection of the kernels of all finite dimensional representations is zero.

The first property is due to Weyl, the second to Harish-Chandra (see [Dix77, 2.5.7, p. 84]).

In the following we consider algebras $A$ over arbitrary fields $K$ which satisfy these two properties. For this purpose we introduce the following condition FCR (= "Finite dimensional representations are Completely Reducible"):

(FCR) The algebra $A$ is residually finite-dimensional and every finite dimensional representation of $A$ is completely reducible. 
Well-known examples of FCR-algebras (in characteristic zero) are the enveloping algebras of semisimple Lie algebras and their factor algebras (and, of course, the finite dimensional semisimple algebras) and the quantum enveloping algebras $\mathfrak{U}_{q}(\mathfrak{g})$ for $q$ not a root of unity. In [KrS94] we showed that one can construct many more such algebras by using the following proposition (loc. cit. Proposition 1).

Proposition 3. Let $R$ be a noetherian $K$-algebra satisfying condition FCR and let $H$ be a finite group of automorphisms of $R$ where $|H|$ and char $K$ are prime. Then the invariant algebra $R^{H}$ also satisfies FCR.

In fact, it is not hard to see that $R$ is a finitely generated $R^{H}$-module. Therefore, the claim is a consequence of the following more general result.

Theorem 3. Let $R$ be a $K$-algebra and $A \subset R$ a subalgebra. Assume that $R$ is finitely generated as an A-left module and that $R=A \oplus V$ where $V$ is an $A$-bimodule. If $R$ satisfies $\mathrm{FCR}$, then so does $A$.

Proof. It is obvious that $A$ is residually finite-dimensional. So it remains to prove that $A / I$ is semisimple for every two sided ideal $I \subset A$ of finite codimension. For this it suffices to show that every left ideal $J / I \subset A / I$ is a direct summand. We have

$$
R I=I \oplus V I \subset R J=J \oplus V J \subset R=A \oplus V
$$

and so

$$
R J / R I=J / I \oplus V J / V I \subset R / R I=A / I \oplus V / V I .
$$

By assmption, $R / R I$ is a finite-dimensional $R$-module and therefore semisimple. Thus the submodule $R J / R I$ is a direct summand. It follows that $J / I$ is a direct summand of $R / R I$ as an $A$-module, hence a direct summand of $A / J$.

\section{References}

[Be71] G. Bergman, Groups acting on hereditary rings, Proc. London Math. Soc. 23 (1971), 70-82.

[Dix77] J. Dixmier, Enveloping algebras, North-Holland Publishing Company, AmsterdamNew York-Oxford, 1977.

[KrS94] H. Kraft, L.W. Small, Invariant algebras and completely reducible representations, Math. Research Letters 1 (1994), 297-307.

[Mo80] S. Montgomery, Fixed rings of finite automorphism groups of associative rings, Lecture Notes in Math. vol. 818, Springer-Verlag, Berlin-Heidelberg-New York, 1980.

[Pi82] R.S. Pierce, Associative algebras, Graduate Texts in Mathematics, vol. 88, SpringerVerlag, Berlin-Heidelberg-New York, 1982.

[St74] R. Steinberg, Conjugacy classes in algebraic groups (2nd enl. ed.), Lecture Notes in Math. vol. 366, Springer-Verlag, New York-Heidelberg-Berlin, 1974.

Mathematisches Institut der Universität BAsel

Rheinsprung 21, CH-4051 BAsEL, SWITZERLAND

E-mail address: kraft@math.unibas.ch

Department of Mathematics, University of California, San Diego

9500 Gilman Drive, La Jolla, CA 92093-0112, USA

E-mail address: lwsmall@ucsd.edu, nwallach@ucsd.edu 